\title{
Responsabilità sociale e gestione etica dell'impresa tra profitto e primato della persona umana ${ }^{1}$
}

\author{
Doc. dr. Ubaldo Comite \\ Organizzazione delle Amministrazioni Pubbliche e \\ Bilancio degli Enti Pubblici \\ Università della Calabria - Facoltà di Economia
}

\section{L'oggetto della Responsabilità Sociale di Impresa}

In ambito imprenditoriale ed aziendalistico si afferma che la Responsabilità Sociale d'Impresa ${ }^{2}$ risponde all'esigenza di integrazione delle preoccupazioni di natura etica all'interno della visione strategica dell'impresa, trattandosi di gestire efficacemente le problematiche d'impatto sociale ed etico nei vari ambiti d'azione imprenditoriale ${ }^{3}$.

Un'impresa che adotti un comportamento socialmente responsabile, monitorando e rispondendo alle aspettative economiche, ambientali, sociali di tutti i portatori di interesse coglie anche l'obiettivo di conseguire un vantaggio competitivo e massimizzare gli utili di lungo periodo ${ }^{4}$. Èquindi, di fondamentale importanza l'attività dedicata al mantenimento delle relazioni con l'esterno, verso i cosiddetti stakeholder.

\footnotetext{
1 Per ulteriori approfondimenti si rimanda alla relazione dell'autore dal titolo: Responsabilità sociale d'impresa ed etica degli affari: la complessità delle motivazioni umane in economia aziendale presentata al Convegno - Giornate di Studio: Filosofia, Cristianesimo e Capitalismo: dal Pensiero Francescano Medievale alla Modernità; 30-31 marzo 2009, Rende (Cs) Italy.

${ }^{2}$ Terzani S., Responsabilità sociale dell'azienda, Rivista Italiana di Ragioneria ed Economia Aziendale n'7-8, Roma, 1984

${ }^{3}$ Un'altra ragione, che traspare dal contesto ambientale, ci viene fornita dall'enfasi con cui L'European multistakeholder forum on CSR mette in risalto, infatti si afferma che la RSI deve diventare un elemento fondamentale della cultura aziendale: "... CSR is embedded in the corporate culture, values and attitudes... this is especially true when CSR is part of the founders and management's values", European Multistakeholder forum on CSR-final risults and recommendations, 29 giugno 2004-Final Report: 4.

${ }^{4}$ Baldarelli M., Le aziende eticamente orientate. Mission, governance, accountability, Clueb, Bologna, 2005

${ }^{5}$ La governance, in poche parole, deve essere orientata al rispetto della giustizia prima che agli scambi equivalenti, enciclica Centesimus Annus di papa Giovanni Paolo II, 1991.
} 
aziendale, l'attenzione agli stakebolder è divenuta di importanza cruciale per le imprese e spesso lo sviluppo nel tempo di relazioni positive con tali soggetti può diventare un elemento di valore aggiunto per l'impresa ${ }^{6}$. Ma il comportamento più o meno etico di un'impresa interessa potenzialmente tutti i cittadini, ai quali non bastano astratte dichiarazioni di principi e valori: essi esigono ormai un impegno quotidiano e credibile, frutto di una precisa politica manageriale e di un sistema aziendale organizzato a tal fine ${ }^{7}$.

La Responsabilità Sociale di Impresa non va confusa con la corporate philantropy", che prevede la "restituzione" di una parte del profitto in fon-

${ }^{6}$ In particolare èinteressante la teoria di Naughton e Alford, che prendono in esame la
teoria degli stakeholders affermando che la stessa è nata in risposta ad un'eccessiva stru-
mentalizzazione dei gruppi di interesse dell'azienda ed è diretta al raggiungimento esclusi-
vo degli obiettivi economici aziendali mentre gli interlocutori dell'azienda sono considerati
mezzi per il raggiungimento dei fini aziendali, Alford H. and Naughton M., Managing as if
Faith mattered. Cristian social principles in the modern organization, Univ. Of Notre Dame
Press, Indiana, 2001. Comunque già lo stesso Besta affermava che la produzione di ricchez-
za da parte dell'azienda è un mezzo per il soddisfacimento dei bisogni, Besta F., La Ragio-
neria, Vol I, II e III, Villardi, Milano.,1916.
${ }^{7}$ Alcuni Autori, tra cui Carroll, affrontano la tematica della responsabilità sociale conside-
randola sullo stesso piano della legge, dell'economia, dell'etica, della socialità e della filan-
tropia, Carrol. B., Corporate social responsibility. Evolution of a definitional costruct, Busi-
ness and society, Sept. 38.3 .1999 . Altri Autori (Bagnoli) fanno prevalere l'approccio indutti-
vo e quindi questo ha una sua valenza, seppur parziale, in quanto occorre precorrere i
tempi da parte dello studioso e capire dove sta andando il mondo attraverso una compren-
sione dei cambiamenti storici e, di conseguenza, dei cambiamenti della cultura aziendale
con tutte le conseguenze che questo comporta, Bagnoli L., Quale responsabilità sociale per
l'impresa?, F. Angeli, Milano, 2004 .
8 ${ }^{8}$ Le attività di Corporate Philanthropy comprendono le modalità con cui imprese e organizzazioni non profit stabiliscono partnership reciprocamente vantaggiose, èuno degli strumenti con cui valutare il Social Commitment dell'impresa nei confronti di uno stakebolder particolare che è la Comunità di appartenenza. Se le più grandi imprese italiane sono consapevoli che alti traguardi competitivi si giocheranno su nuovi elementi non direttamente correlati alle dinamiche economiche e quindi sarà fondamentale ridisegnare un nuovo ruolo all'interno dell'ambiente di riferimento, tuttavia la maggior parte delle piccole-medie imprese, sono ancora orientate ad una Philanthropy di beneficenza o a politiche di charity strumentali dettate da necessità contingenti, dove l'attivit à di Giving dell'impresa è distaccata dal suo processo di business. Oggi dove la ricchezza e concorrenzialità di un'impresa dipende sempre di più dai suoi asset intangibili (da una recente indagine-tratta dal saggio"Philanthropy and Business: The Changing Agenda", redatto da Sophia A. Muirhead della associazione The Conference Board nel Maggio 2006più di un terzo delle aziende intervistate hanno dichiarato che nel 2006 "misurare i risultati" è la più grossa sfida che si pone nell'affrontare programmi di donazioni aziendali) diventa fondamentale introdurre metodologie di valutazione dei benefici intangibili derivanti dalle pratiche di giving dell'impresa. Per un loro consolidamento delle attività di giving nell'impresa ènecessario non solo una loro migliore gestione, ma anche offrire alle imprese nuove modalità strategiche di realizzazione di partnership con le istituzioni non profit, che si strutturino in programmi sistematici, innovativi, capaci di offrire un valore aggiunto visibile alle funzioni fondamentali dell'impresa, come la gestione del Marketing e delle Risorse Umane. Un esempio è costituito dall'area dell'Employee Support e un numero 
do a un processo di massimizzazione dello stesso che può essere di qualsiasi natura. Un passo intermedio è costruito dal sorgere della filantropia sociale che ha come scopo quello di assistere le maestranze e le loro famiglie lungo il corso della loro vita, questo in genere attraverso lo strumento delle Fondazioni. L'evoluzione successiva ci porta all'Impresa Socialmente Responsabile?

Tra gli obiettivi principali che essa persegue:

- un'organizzazione post-tayloristica del lavoro;

- il democratic stakeholding;

- il coinvolgimento attivo dell'impresa nello sviluppo del suo territorio di riferimento.

- Un'organizzazione post-tayloristica del lavoro

Laddove nell'impresa tayloristica il coordinamento era realizzato dalla gerarchia, ora occorre affidarsi all'interdipendenza: il comportamento di ciascun membro dell'organizzazione dipende anche dalle sue aspettative circa le intenzioni e il comportamento effettivo degli altri.

Il meccanismo degli incentivi, per assicurarsi la lealtà e la collaborazione del dipendente, alla lunga si mostra controproducente in quanto si ha il paradosso che, se questi è pagato per essere onesto e leale sul lavoro, si ottiene l'effetto di erodere, nel tempo, la forza delle motivazioni intrinseche degli agenti.

Le imprese socialmente responsabili sono quelle che più efficacemente riescono a utilizzare, a proprio vantaggio, il meccanismo della persuasione nei confronti di tutti coloro che con essa intrattengono rapporti che devono essere improntati a canoni di equità: il dipendente che è persuaso di essere trattato equamente tenderà a comportarsi con diligenza.

\section{- Il democratic stakebolding}

L'idea di base è quella di consentire a tutti coloro che intrattengono rapporti con l'impresa, la possibilità reale di partecipare, in qualche modo $\mathrm{e}$ in qualche forma, al processo decisionale.

Si tratta di passare dallo stakebolder management alla stakebolder democracy, un modello di governance in cui sono gli stessi portatori di inte-

\footnotetext{
sempre maggiore di imprese ha stabilito al suo interno programmi di Matching Gifts per propri dipendenti. Un aspetto innovativo dei programmi di matching gift è il loro connubio con i programmi di Corporate Voluteering, dove l'impresa attraverso una comunicazione istituzionale invita i suoi dipendenti a dedicare alcune ore di lavoro retribuite all'attività di volontariato. Perché queste pratiche si consolidino dovranno diventare uno strumento integrante la mission aziendale, le imprese dovranno essere capaci di allineare i loro programmi di investimento nella Comunità agli obiettivi aziendale e necessariamente organizzare queste attività in strutture definite.

9 Molteni M., Responsabilità sociale dell'impresa. Verso la sintesi socio-competitiva, Vita e Pensiero, Milano, 2004
} 
ressi che, in quanto partner dell'impresa, condividono diritti e doveri. La difficoltà più seria in questo campo riguarda le modalità di rappresentanza di platee molto vaste, basti pensare, ad esempio, all'insieme dei clienti di una grande azienda ${ }^{10}$.

- Il coinvolgimento attivo dell'impresa nello sviluppo del suo territorio di riferimento

Se il territorio non è in grado di assicurare adeguati livelli di istruzione, servizi sanitari in linea con gli sviluppi delle tecnologie bio - medicali, forme assistenziali innovative per la famiglia, ecc, l'impresa porterà delle conseguenze dannose nel perseguire i propri obiettivi. L'impresa socialmente responsabile non può pensare di disinteressarsi a problemi del genere, ritenendo che alla soluzione di questi debba provvedere l'ente pubblico.

\section{Responsabilità sociale e competitività}

La Responsabilità Sociale di Impresa consente all'impresa di far circolare, nel massimo grado possibile, la conoscenza tacita (tacit knowledge) che completa, non si contrappone, alla codified knowledge che è quella che può essere veicolata, e quindi trasmessa da persona a persona o da reparto a reparto, a mezzo di codici ovvero protocolli ${ }^{11}$.

${ }^{10}$ Zamagni S., Responsabilità Sociale delle Imprese e Democratic Stakeholding, Working paper n²8-2006, Facoltà di Economia, Università di Bologna-Forlì

${ }^{11}$ La conoscenza può essere classificata in due categorie: esplicita e tacita. La prima comprende tutto quanto è esprimibile attraverso sistemi formali di comunicazione ed è trasmessa per mezzo di libri, manuali o corsi, in quanto ha un contenuto prevalentemente logico e linguistico; la conoscenza esplicita è la base della cultura occidentale. La seconda è invece il risultato di processi non formali né logici, ed è composta da intuizioni, nozioni personali, esperienza, cultura o valori morali; può essere trasmessa per mezzo di metafore e analogie, o con l'esempio pratico. La conoscenza tacita si può dividere a sua volta in sottocategorie; la divisione più semplice è tra conoscenza tecnica e cognitiva: la prima riguarda la manualit à e si colloca nel campo delle arti e delle abilit à concrete; la seconda concerne l'elaborazione e coinvolge i modelli mentali, gli schemi, i paradigmi, le prospettive che ciascuno di noi crea. Anche se talvolta si confonde l'acquisizione di conoscenza già esistente con il processo di creazione e di sviluppo, quest'ultimoè sostanzialmente diverso, e nasce dall'apprendimento inteso come capacità di ottenere know-how per risolvere problemi specifici in base al contesto in cui si deve operare, alla quale segue la capacità di cambiare il contesto stesso. Considerando che gli apprendimenti più profondi nascono da esperienze dirette, è evidente il forte legame tra apprendimento e conoscenza tacita; si deve convenire quindi che la maggior parte della conoscenza è di tipo tacito.

La conoscenza tacita viene anche indicata come esperienzale (corporea), simultanea ("qui e adesso") e analogica (pratica), mentre la conoscenza esplicita è anche razionale (mentale), sequenziale ("là e allora") e digitale (teorica). Il passaggio di conoscenza da un tipo all'altro, o all'interno dello stesso tipo, prende il nome di conversione, e ogni possibile conversione crea nuova conoscenza con un contenuto ben preciso. Il processo ha avvio dalla costruzione di un campo di interazione, nel quale sia possibile avviare un processo di condivisione di conoscenza e di modelli mentali: questa conversione prende il nome di socializzazione e riguarda la conversione di conoscenza tacita in se stessa. Il dialogo basato su metafore e analogie permette la creazione di conoscenza esplicita: questa conversione 
La conoscenza tacita, invece, è quella che alberga nella mente delle singole persone e che non può essere trasmessa ad altri se non con il loro deliberato consenso ${ }^{12}$.

E' dimostrato che il successo nella moderna impresa post - taylorista dipende assai più dalla sua capacità di estrarre conoscenza tacita da tutti i suoi collaboratori che non dalla sua abilità di veicolare la conoscenza codificata.

Ancora una volta per "comprare" la conoscenza tacita non sono sufficienti gli incentivi economici. Infatti, uno schema di incentivo nasconde sempre una relazione di potere.

Con la "persuasione" che ciò corrisponde al suo proprio bene il lavoratore può essere naturalmente indotto a mettere in circolo il suo patrimonio di conoscenze. Ovviamente politiche aziendali contrarie alla dignità dei lavoratori (ad esempio esercizio di mobbing) o politiche di Responsabilit à Sociale di Impresa enunciate ma non realizzate neutralizzano sul nascere qualsiasi ipotesi di scambio tacit knowledge.

Vi è anche un secondo argomento importante a difesa della tesi per cui la Responsabilità Sociale di Impresa è una strategia sostenibile per l'impresa che decide di porla in pratica: la responsabilità sociale del consumatore.

Si sta ormai affermando il superamento del modello del consumatore come ricettore passivo delle proposte di acquisto che va cedendo il passo al consumatore critico, o etico, che intende contribuire a "costruire" l'offerta di quei beni ${ }^{13}$. Numerose ricerche dimostrano che fino all' $80 \%$ dei consumatori europei si dichiara propenso a favorire lo sviluppo di imprese impegnate seriamente sul fronte della Responsabilità Sociale di Impresa ${ }^{14}$.

Il boom dei fondi etici e della cosiddetta finanza etica per un verso, e la crescita inaspettata del commercio equo e solidale confermano appieno questi dati statistici.

Se l'obiettivo da perseguire è quello di come giungere a un ordine sociale più avanzato sotto il profilo della democrazia e della libertà, allora il consumo non può non configurarsi come fattore di civilizzazione, evol-

\footnotetext{
prende il nome di esteriorizzazione. La conoscenza esplicita così creata origina prodotti, servizi o soluzioni innovative, formando altra conoscenza esplicita attraverso la combinazione. La produzione di questi beni genera nuova esperienza che accresce il bagaglio di conoscenza implicita: è l'interiorizzazione. A questo punto il ciclo ricomincia, in una spirale senza fine, e il risultato di queste trasformazioni è l'innovazione contenuta nei nuovi prodotti e la spinta alla creazione di ulteriore conoscenza.

12 Payne D.M., Raiborn C.A., Sustainable Development: The ethics support the economics, Journal of Business Ethics, 32, 2001

${ }_{13}$ Paradigmatico èil caso della Nike travolta dallo scandalo del lavoro minorile e sottopagato in India.

${ }^{14}$ Ministero del Lavoro e delle Politiche Sociali, Roma, 2007
} 
vendo verso forme culturalmente sempre più ricche: la Responsabilità Sociale di Impresa rappresenta una delle forme più avanzate di innovazione ${ }^{15}$.

A supporto di ciò si può leggere: "L'implementazione, quindi, della responsabilità sociale come mission specifica o come strumento di governance nelle odierne imprese lucrative, in conclusione, è e sarà sempre più strettamente connessa da un canto all'esigenza dell'impresa di non causare danni e dall'altro al diritto / dovere di informare gli interlocutori sociali rilevanti sull'eticità del suo comportamento gestionale, al fine di garantirsi così la legittimazione all'agire ed il consenso all'agire da parte della società civile $^{16}$. Inoltre, sembra importante sottolineare quanto scrive Ponzanelli in merito al concetto di impresa sociale: "Non può essere enunciato un giudizio di piena soddisfazione degli obiettivi dell'impresa sociale per la sola constatazione che essa riesca a mantenere integro ed a retribuire il capitale, ma altresìper la constatazione che chi ne è responsabile riesce a fondere i bisogni e le soddisfazioni di ogni partecipante coi bisogni dell' impresa"17.

\section{Alcune critiche rivolte alla Responsabilità Sociale di Impresa}

La principale critica che si muove alla Responsabilità Sociale di Impresa è che questa è una operazione di mera cosmesi finalizzata a dare alle imprese la maschera del volto umano, un paravento per consentire a imprese senza scrupoli morali di eliminare dal mercato i propri rivali o di ridurne la forza competitiva ${ }^{18}$.

La seconda è legata alla prima e si poggia sulla paura che i grandi gruppi, investendo quote per loro anche marginali, possano "comperarsi" la reputazione ritenuta necessaria, marginalizzando quindi le imprese più piccole e meno visibili al grande pubblico salvo mutare strategia quando il contesto competitivo diventasse particolarmente severo. Ma in un mercato evoluto come quello attuale queste sarebbero solo strategie diversive che avrebbero sicuramente vita breve.

Al management dell'impresa "tradizionale" nessuno chiederà mai di dare conto del valore espressivo da essa generato; solo il manager dell'impresa socialmente responsabile potrà dare evidenza del valore aggiunto sociale che la sua azione genera ${ }^{19}$.

\footnotetext{
${ }^{15}$ Cafferata R., Andamento ed evoluzione del rapporto tra impresa e ambiente, Impresa Progetto, 2005.

${ }^{16}$ Matacena A., L'accountability nelle imprese lucrative e sociali. Verso una possibile convergenza?, Intervento al convegno: La responsabilità sociale delle imprese tra evoluzione scientifica e processi attuativi, Catania: 8-9 settembre 2005.

${ }_{17}^{17}$ Ponzanelli G., Impresa e ambiente, Circolo giuridico dell'università, Siena, 1963.

${ }^{18}$ Gregoratti C., Prospettive critiche in materia di RSI e sviluppo. Una risposta, University of Manchester, 2006

${ }^{19}$ Rusconi G., Il bilancio sociale. Economia,etica e responsabilità d'impresa, Ediesse, Roma, 2006
} 
Perché la Responsabilità Sociale di Impresa produce non solamente un valore strumentale, ma anche un valore espressivo che è certamente misurabile, sia pure in modo differente da quello con cui si misura il valore strumentale dell'impresa, questo perché vi sono agenti che hanno preferenze etiche - agenti cioè che attribuiscono valore al fatto che l'impresa pratichi la Responsabilità Sociale di Impresa indipendentemente dal vantaggio materiale che a essi può derivarne ${ }^{20}$.

\section{Etica, il valore aggiunto d'impresa}

Il tema di un'impresa etica si colloca all'interno di questa vasta problematica che, individua lo sviluppo sostenibile e l'importanza dell'opinione pubblica, quali elementi fondamentali nella definizione dell'ambiente competitivo e del "valore aggiunto" dell'impresa ${ }^{21}$.

Parte del mondo imprenditoriale, ancora oggi, si interroga se il perseguimento di finalità sociali, comportando un aumento dei costi, è da considerare un fattore di "svantaggio competitivo" o se, per contro, il raggiungimento di un ottimale livello di efficienza e di rendimento economico non possa più prescindere dalla programmazione ed attuazione, all'interno dell'impresa stessa, anche di pratiche che sottolineano la propria funzione sociale, tra le quali la valorizzazione, la protezione e lo sviluppo delle risorse umane in essa impiegate, la previsione e il rispetto di solidi principi a tutela della salute e della sicurezza dei consumatori, la salvaguardia dell'ambiente esterno, l'integrazione dell'impresa nella comunit à locale, garantendo uno sviluppo, socialmente valido, del progresso economico ${ }^{22}$. Un dubbio che appare non tenere conto dei radicali cambiamenti che stanno caratterizzando il nuovo scenario economico mondiale e dei risultati particolarmente positivi, in termini economici e di immagine, ottenuti da imprese che per prime hanno intrapreso la via dello sviluppo sostenibile. Altrettanto numerosi sono gli esempi di imprese che tralasciando di considerare l'importanza che gli aspetti etici e morali rivestono nel giudizio del consumatore, hanno dovuto far fronte a sensibili cali di immagine, perdite di quote di mercato e di volumi delle vendite ${ }^{23}$.

\footnotetext{
${ }^{20}$ Di Toro P., Governance, etica e controllo, Cedam, Padova, 2000

${ }^{21}$ A supporto di ciò, secondo Zadeck l'etica sta assumendo rilevanza proprio in relazione al cambiamento che viene effettuato nel contesto ambientale in cui l'azienda opera: " the argument that "relationship" count is of course not new. What is new, however, are the economic and social conditions under which this most recent cycle of thinking and practice is happening", Zadek S., Balancing performance, ethics, and accountability, Journal of business ethics, 17, 1998

${ }^{22}$ Beckett C., Maynard A., Values E Ethics in Social Work, Sage Publication Ltd, London, 2005

${ }^{23}$ Catturi G., Intervento, in Riccaboni A., (a cura di), Etica ed obiettivi di impresa, Dipartimento di Studi Aziendali e Sociali, Siena, 1995
} 
Il giudizio del consumatore, oggi, infatti, va ben oltre il rapporto costi - benefici orientando le scelte verso quelle imprese di cui condivide valori e principi e che sul piano concreto realizzano prodotti e processi rispettosi dell'ambiente e dell'elevata qualità; non discriminano i dipendenti in base al sesso, razza, religione e appartenenza politica; non testa i prodotti sugli animali; hanno a cuore diritti umani e l'equa distribuzione della ricchezza nel mondo; si impegnano nella riduzione di problemi sociali, quali la criminalità, l'analfabetismo, la povertà.

I consumatori, infatti, tendono a premiare le imprese che si manifestano particolarmente attente a fattori quali il commercio equo e solidale, la razionalizzazione delle risorse primarie, il rispetto dell'ambiente e della persona.

In Italia, circa il 64\% della popolazione ritiene che il mondo economico non dedichi sufficiente attenzione al concetto di etica d'impresa, e tra questi circa il 20\% considera molto importante nella scelta dei propri acquisti l'impegno e la responsabilità sociale dell'azienda produttrice, dichiarandosi disponibili ad accettare un prezzo più alto del bene stesso ${ }^{24}$.

Se per i manager, dunque, l'etica d'impresa rappresenta un importante strumento di governo dell'impresa, per migliorare le performance finanziarie, i processi di aggregazione interni e la gestione operativa, per i cittadini e per i consumatori è la nuova rappresentazione del valore aggiunto dell'impresa: la reputazione ${ }^{25}$.

\section{La reputazione dell'impresa}

La reputazione dell'impresa, fonda le sue radici su principi e regole di comportamento preventivo che rafforzano la fiducia e la cooperazione dei suoi stakeholder, favorendo nuovi rapporti commerciali, transazioni continue, riduzioni dei costi di controllo, gestione e di contrattazione, incrementi dell'efficienza aziendale e dei profitti ${ }^{26}$. La coscienza etica è divenuta il principale elemento di qualità dell'impresa, ma affinchè rappresenti un effettivo punto di forza, è doveroso che divenga parte integrante della strategia di base dell'azienda, interagente con tutti i campi della gestione aziendale: finanza, produzione, marketing, risorse umane ${ }^{27}$.

\footnotetext{
${ }^{24}$ Istituto Italiano di Statistica, Roma, 2008

25 Coda ribadisce che l'impresa adeguata ai tempi moderni, è quella di utilizzare l'etica come parte integrante dell'obiettivo e non quella di utilizzare l'etica semplicemente come un vincolo. Infatti,"A ben vedere questa seconda opzione, a differenza della prima, richiede ai vertici aziendali, non solo di prendere fermamente le distanze da qualsiasi comportamento contrario all'etica, ma anche di fare una decisa scelta di campo in favore della concezione che vede nell'impresa un public good, da gestire comunque responsabilmente nei confronti di tutti gli stakeholders con l'obiettivo di creare valore per tutti", Coda V., Responsabilità sociale e strategica dell'impresa, in Sacconi G., Responsabilità sociale e governo d'impresa. Problemi, teorie e applicazioni. Un esame critico, Maggioli, Rimini. 2005.

${ }^{26}$ AA.VV., Teoria degli stakeholder, F. Angeli, Milano, 2007

${ }^{27}$ Di Giandomenico M., Il bilancio sociale e il modulo aziendale etico, GiuffrèMilano, 2008.
} 
Fondamentale in tale ottica è il coinvolgimento di tutte le parti interessate nella condivisione della mission aziendale e nella individuazione dei bisogni e delle esigenze di tutti gli stakeholder. clienti, fornitori, dipendenti, soci, finanziatori, enti pubblici, comunità e ambiente, garantendo un elevato grado di coesione, basato sulla correttezza e trasparenza delle relazioni e sul rispetto reciproco ${ }^{28}$.

La puntualità negli ordinativi, il rispetto dei termini di pagamento, l'abbandono di pratiche di autofinanziamento sono misure che fidelizzano il fornitore, rafforzandone fiducia e considerazione, e favoriscono prezzi vantaggiosi.

Il cliente, per restare tale a lungo, deve ricordare nel tempo, l'azienda, per la professionalità dimostrata, la cortesia, la disponibilità e l'assenza di mediocrità in tutte le fasi del processo produttivo. La sua soddisfazione richiede la fornitura di servizi efficienti ed aggiuntivi, quali l'assistenza, la consulenza, la manutenzione, lo scambio informativo, da attuarsi in maniera impeccabile, nel pieno rispetto dell'ambiente circostante.

Il miglior rapporto con $\mathrm{i}$ clienti, $\mathrm{i}$ fornitori e le istituzioni pone l'azienda in una posizione di vantaggio competitivo, in termini di riduzione di costi, tempi di approvvigionamento, qualità dei prodotti, sistemi di controllo e sicurezza del prodotto e di maggiori volumi dei ricavi.

Il massimo coinvolgimento dei lavoratori, accresce l'orgoglio aziendale e l'impegno personale, crea un ambiente di lavoro migliore, sereno e più sicuro.

Una organizzazione che favorisca stabilmente la delega, la cooperazione, la partecipazione porta allo sviluppo di un contesto lavorativo in cui l'autonomia e la collaborazione prevalgono sulla strutturazione, la formalizzazione, il controllo gerarchico e in cui si riduce l'assenteismo, la conflittualità e l'incomprensione, aumentando la produttività e la correlata qualità produttiva ${ }^{29}$.

\section{Creazione del profitto e primato della persona umana}

Coloro che aderiscono all'idea che l'esercizio dell'impresa debba essere considerato un mezzo per creare e diffondere il benessere, sono profondamente convinti che sia necessario coniugare due valori assolutamente fondamentali: a) il ruolo sociale dell'impresa: creare profitto (ricchezza,

\footnotetext{
${ }^{28}$ Zadra G., Il rendiconto agli stakeholder. Una guida per le banche, http://www.abi.it/doc /home/conoscereBanche/FinanzaEtica/doc/tmp1163169967596_30 Rendiconto.pdf.

29 Catturi G., L'azienda universale. L'idea forza, la morfologia e la fisiologia, Cedam, Padova, 2003
} 
benessere, valore aggiunto) tramite la produzione di beni e servizi utili; b) il primato della persona umana, anche nell'ambito economico lavorativo ${ }^{30}$.

Nella società industriale, per lungo tempo, c'è stata una certa difficoltà a coniugare tali principi, la Chiesa stessa ha guardato con pregiudizio all'impresa, a causa del modo con cui il profitto veniva - e spesso viene ancora - perseguito in modo spregiudicato.

Per contro, sul fronte imprenditoriale la centralità della persona umana era vista come principio velleitario.

Oggi questi valori sono maggiormente condivisi; nel perseguire il profitto, è chiesto all'impresa un comportamento etico (si parla di business ethics), ciò̀ un comportamento virtuoso per cui: il profitto è considerato un indicatore importante, ma non l'indicatore per eccellenza, e il suo perseguimento deve avvenire in un contesto di sostenibilità sociale ed ambientale ${ }^{31}$.

E' significativo un documento del magistero ecclesiastico della Chiesa di Roma, che non soltanto riassume bene i concetti sopra esposti, ma che espone con molta chiarezza un pensiero che oggi è assolutamente condiviso, in quanto esprime valori profondamente umani, la lettera enciclica Centesimus Annus di papa Giovanni Paolo II (1991): la Chiesa riconosce la giusta funzione del profitto, come indicatore del buon andamento dell'azienda. Quando un'azienda produce profitto, ciò significa che i fattori produttivi sono stati adeguatamente impiegati e i corrispettivi bisogni umani debitamente soddisfatti. Tuttavia, il profitto non è l'unico indice delle condizioni dell'azienda. E' possibile che i conti economici siano in ordine e, insieme, che gli uomini, che costituiscono il patrimonio più prezioso dell'azienda, siano umiliati e offesi nella loro dignità. Oltre ad essere moralmente inammissibile, ciò non può non avere, in prospettiva, riflessi negativi anche per l'efficienza economica della struttura imprenditoriale. Scopo dell'impresa, infatti, non è semplicemente la produzione del profitto, bensì l'esistenza stessa dell'impresa come comunità di uomini che, in diverso modo, perseguono il soddisfacimento dei loro fondamentali bisogni e costituiscono un particolare gruppo al servizio dell'intera società.

Il profitto è un regolatore della vita aziendale, ma non è l'unico; ad esso va aggiunta la considerazione di altri fattori umani e morali che, nel lungo periodo, sono almeno egualmente essenziali per la vita dell'impresa ${ }^{32}$.

\footnotetext{
${ }^{30}$ Farneti G., in AA.VV., Il business alla ricerca dell'etica. Fondazioni di impresa $e$ responsabilità sociale delle aziende. Atti del Convegno. Philanthropy Centro Studi, Facoltà di Economia, Forlì, 2004

${ }^{31}$ Corno F., L'etica nel governo dell'impresa. Convergenza tra pensiero laico e Dottrina Sociale, Guerini e Associati, Milano, 2002

${ }^{32}$ Andreaus M., Credibilità sociale e credibilità finanziaria, in Finanza e industria in Italia, Atti del XXIX Convegno AIDEA, Il Mulino, Bologna, 2007
} 
Bench è l'attenzione all'eticità dei comportamenti, nel campo dell'attività economica sia connaturata all'agire sociale (fin dal momento stesso in cui l'uomo esce dall'ambito familiare per operare sul mercato), con l'età industriale, prima, e con la globalizzazione poi, l'attenzione all'eticità di questi comportamenti assume nuove connotazioni. Nel caso della business ethics si tratta di una presa di coscienza che è cresciuta con progressione senza avere specifici legami con persone o specifici accadimenti, ma quasi sempre, purtroppo, come reazione a scandali che si ripropongono con drammatica costanza, quali sfruttamento del lavoro di bambini nei Paesi poveri, corruzione, concussione, insider trading, speculazioni, pratiche disoneste.

A partire dagli anni Ottanta in tutti i settori industriali si è assistito a un profondo e generale cambiamento, che ha riguardato molteplici aspetti fortemente collegati tra loro, al punto che non è semplice riuscire a separarli dandone una spiegazione autonoma:

1) per le imprese sono affiorate nuove esigenze, sintetizzabili in termini di flessibilità, snellezza strutturale, qualità, efficienza, che hanno richiesto adeguate risposte (azienda corta, azienda rete, terziarizzazione, outsourcing);

2) si è verificata una evoluzione nelle caratteristiche e nei comportamenti dei clienti - consumatori, con un aumentata presa di coscienza del loro reale potere contrattuale;

3) la società civile, con la presa di coscienza di essere soggetto attivo ha, verso il sistema imprese, un ventaglio più vasto e articolato di esigenze: ad esse viene chiesto maggiormente di fare la "cosa giusta" (the right thing), non semplicemente "cose giuste" (right things). La nuova strategia che l'impresa deve adottare per essere in grado di rispondere e far fronte a pressioni sociali internazionali è la C.S.R. (con termine inglese Corporate Social Responsibility) o R.S.I. (con termine italiano Responsabilità Sociale di Impresa).

L'etica di cui si sente il bisogno non è quella che pone vincoli o proibizioni, ma che offre criteri e orientamenti in vista del bene delle persone, nelle dimensioni individuali e comunitarie; si potrebbe definire come un'esigenza di maggior umanizzazione. Cresce, quindi, l'esigenza di un'economia che offra altri criteri, oltre a quelli della crescita del Prodotto Interno Lordo (P.I.L.) (a livello macroeconomico) e della massimizzazione del profitto (a livello microeconomico).

E' una vera e propria cultura d'impresa orientata verso la trasparenza e l'acquisizione di una coscienza fondata sulla necessità di far interagire al meglio gli interessi economici delle singole imprese con quelli sociali e ambientali (impatto ambientale e sviluppo sostenibile) del contesto di riferimento. Sono i temi della responsabilità sociale. 
Gli imprenditori - dirigenti - professionisti che hanno accolto queste tematiche vi si riconoscono, con una forte tensione a un impegno etico personale, mettendo l'accento sulla necessit à di una responsabilit à imprenditoriale finalizzata alla costruzione del bene comune.

Nell'etica cristiana il bene utile, il bene giusto e il bene bello devono essere coniugati insieme per il bene di tutti.

La responsabilit à della costruzione del bene comune non deve più spettare soltanto allo Stato, ma a tutti i soggetti che operano nella società secondo il fondamentale principio della sussidiarietà ${ }^{33}$.

\footnotetext{
${ }^{33} \mathrm{Il}$ principio di sussidiarietà riguarda i rapporti tra stato e società e rappresenta un fondamentale principio di libertà e di democrazia. Il suo contenuto si individua attraverso le tre seguenti affermazioni:
}

a) non faccia lo Stato ciò che i cittadini possono fare da soli;

b) lo Stato intervenga solo quando i singoli e i gruppi che compongono la società non sono in grado di farcela da soli;

c) l'intervento sussidiario dello Stato e delle sue articolazioni deve comunque essere portato al livello più vicino al cittadino.

Tale principio implica quindi che:

a) le diverse istituzioni, nazionali come sovranazionali, debbono tendere a creare le condizioni che permettono alla persona e alle aggregazioni sociali (i cosiddetti corpi intermedi: famiglia, associazioni, partiti, ecc.) di agire liberamente senza sostituirsi ad essi nello svolgimento delle loro attività se sono in grado di agire per proprio conto;

b) l'intervento dell'entit à di livello superiore deve essere temporaneo e teso a restituire l'autonomia d'azione all'entità di livello inferiore;

c) l'intervento pubblico deve essere attuato quanto più vicino possibile al cittadino garantendo così la prossimità del livello decisionale a quello di attuazione

In ambito giuridico si parla di sussidiariet à verticale quando i bisogni dei cittadini sono soddisfatti dall'azione degli enti amministrativi pubblici e di sussidiarietà orizzontale quando tali bisogni sono soddisfatti dai cittadini stessi, magari in forma associata elo volontaristica. Infine occorre ricordare che il principio di sussidiarietà:

- é uno dei fondamenti della Dottrina Sociale della Chiesa e la formulazione originaria si trova nell'enciclica Quadrigesimo Anno (1931) di papa Pio XI (" (..) siccome non é lecito togliere agli individui ciò che essi possono compiere con le loro forze e l'industria propria per affidarlo alla comunità, cosî é ingiusto rimettere ad una maggiore e più alta società quello che dalle minori e inferiori comunit si può fare. Ne deriverebbe un grave danno e uno sconvolgimento del retto ordine della societ à poiché l'oggetto naturale di qualsiasi intervento della società stessa é quello di aiutare in maniera suppletiva (subsidium afferre) le membra del corpo sociale, non già distruggerle ed assorbirle.");

- è stato sancito dall'Articolo 5 del Trattato CEE che richiama la sussidiarietà come principio regolatore dei rapporti tra Unione e stati membri;

- è stato recepito nell'ordinamento italiano con l'art. 118 della Costituzione.

Sull'argomento si vedano, tra gli altri, Millon -Delsol C., Il principio di sussidiarietà, Giuffrè Milano, 2003 e Donati P., Colozzi I. (a cura di), La sussidiarietà: che cos'è e come funziona, Carocci, Roma, 2005.

Per ben comprendere l'enfasi data al principio di sussidiarietà si veda anche Antonini L., Il principio di sussidiarietà: un fondamento del nuovo Welfare, in, Areté, n. 1. 2008, Maggioli, Rimini, 2008. Proprio all'inizio del lavoro l'Autore ricorda che la Costituzione Italiana, con la modifica del 2001, è la prima che contempla espressamente il principio di sussidiarietà orizzontale. 
Si passa così dal Welfare State al Welfare Society ${ }^{34}$. Il perseguimento del profitto tende a collocarsi in un quadro più generale di "creazione di valore" per tutti i soggetti che direttamente o indirettamente sono associati all'impresa (c.d. patto sociale).

\section{Considerazioni conclusive}

E' importante che si diffonda la cultura della Responsabilit à Sociale di Impresa; non solo per un puro impulso filantropico, ma anche per lo sviluppo dell'economia. L'impresa opera nell'ambiente e, pertanto, dovrebbe realizzarsi un'osmosi per la quale non è solo l'ambiente ad influenzare le scelte dell'impresa ma è anche l'impresa che, con le sue scelte, condiziona l'ambiente.

Una migliore qualità della vita, una effettiva tutela della salute e della sicurezza sul lavoro, una maggiore partecipazione nelle scelte economiche all'interno della comunità in cui si vive sono le aspettative del cittadino del XXI secolo, che l'impresa non può ignorare se vuole continuare a crescere. L'apertura sociale è per questo non soltanto un bene, ma anche una vera e propria necessit à. Questa può addirittura divenire un vantaggio competitivo in quanto l'attenzione rivolta dal consumatore all'impatto ambientale della gestione e dei prodotti possono determinare la scelta di una impresa rispetto ad un'altra.

E' strabiliante come questo principio, già esposto con lucidità nell'enciclica Rerum Novarum (papa Leone XIII -1891) ed enunciato formalmente nell'enciclica Quadragesimo Anno (papa Pio XI - 1931), fosse di fatto sconosciuto fino al Trattato di Maastricht del 1992 (preambolo e art. 3B, nuova formulazione dell'art. 118 della Costituzione Italiana) e sia diventato cultura condivisa in pochissimi anni.

Per un approfondimento ulteriore sul tema, si veda ex aliis Comite U., Creazione di valore e sussidiarietà nell'amministrazione pubblica: un approccio economico-aziendale in G.P. Calabrò (a cura di), La nozione di sussidiarietà tra teoria e prassi, Edizioni Scientifiche Italiane, Napoli, 2009.

${ }^{34}$ Lo Stato sociale o Stato assistenziale, conosciuto anche come welfare state (stato di benessere tradotto letteralmente dall'inglese), è un sistema di norme con il quale lo Stato cerca di eliminare le diseguaglianze sociali ed economiche fra i cittadini, aiutando in particolar modo i ceti meno benestanti.

La Welfare society si potrebbe anche definire come la società della libera scelta. Una societ à dal potere diffuso dove la responsabilità della gestione sociale, superando l'antica totale delega del cittadino allo Stato Padre-Padrone, è affidata ai corpi intermedi della comunità, responsabilizzati rispetto alla sovranità dei loro beni. Una società nella quale il livello privato e il livello statale cooperino e competano nell'offerta di servizi formando, insieme, un unico sistema pubblico all'interno del quale viva la più plurale e libera scelta dei cittadini. Una società, insomma, che dilati in misura esponenziale la libertà dei singoli e delle famiglie di orientarsi autonomamente, tra diverse "offerte pubbliche", riguardo alle scelte fondamentali della propria esistenza. Il che vuol dire un significativo passo indietro dello Stato nella padronanza monopolistica della nostra vita quotidiana e un parallelo incremento della gestione di beni e servizi da parte dei cittadini. Non meno Stato più mercato, dunque, come si diceva negli anni Ottanta: ma Stato necessario e società responsabile. 
La performance degli alti profitti non basta più la nuova frontiera con cui le aziende devono misurarsi è la efficienza considerata anche dal punto di vista della responsabilità sociale.

Questa strategia deve essere supportata da "valori", da profonde motivazioni, a partire dall'imprenditore e dal management, da condividere e da trasmettere.

Interrogarsi sui "valori dell'impresa" costituisce il passaggio chiave, tutto si gioca sui valori.

Se la conoscenza diventa materia prima, se da un modello "materiale" dove l'attenzione era incentrata sull'ottimizzare comportamenti umani nell'impiego di risorse "limitate", si passa a un modello immateriale dove la conoscenza è risorsa infinita, la persona umana diventa centrale.

Occorre sottolineare, da ultimo, come i "valori", anche nell'impresa, sono tali soltanto se sono condivisi e quindi in grado di motivare a una libera adesione.

Rispondere alle esigenze morali più profonde della persona, ha anche importanti e benefiche ricadute sul piano economico ${ }^{35}$.

Dunque, il primato della persona umana e i "valori" nell'impresa sono i capisaldi su cui costruire lo sviluppo, a dai quali occorre ripartire per uscire dalla crisi attuale.

\section{Bibliografia}

Alford H. and Naughton M., Managing as if Faith mattered. Cristian social principles in the modern organization, Univ. Of Notre Dame Press, Indiana, 2001.

AA.VV., Teoria degli stakeholder, F. Angeli, Milano, 2007.

Andreaus M., Credibilità sociale e credibilità finanziaria, in Finanza e industria in Italia, Atti del XXIX Convegno AIDEA, Il Mulino, Bologna, 2007.

Antonini L., Il principio di sussidiarietà: un fondamento del nuovo Welfare, in, Areté, n. 1. 2008, Maggioli, Rimini, 2008.

Bagnoli L., Quale responsabilità sociale per l'impresa?, F. Angeli, Milano, 2004.

Baldarelli M., Le aziende eticamente orientate. Mission, governance, accountability, Clueb, Bologna, 2005.

Beckett C., Maynard A., Values \& Ethics in Social Work, Sage Publication Ltd, London, 2005.

35 E' questa la "grammatica economica" della Chiesa per il Terzo Millennio, contenuta nell'Enciclica Caritas in Veritate scritta da papa Benedetto XVI (2009). 
Besta F., La Ragioneria, Vol I, II e III, Villardi, Milano, 1916.Cafferata R., Andamento ed evoluzione del rapporto tra impresa e ambiente, Impresa Progetto, 2005.

Carrol. B., Corporate social responsibility. Evolution of a definitional costruct, Business and society, Sept. 38.3.1999.

Catturi G., Intervento, in Riccaboni A., (a cura di), Etica ed obiettivi di impresa, Dipartimento di Studi Aziendali e Sociali, Siena, 1995.

Catturi G., L'azienda universale. L'idea forza, la morfologia e la fisiologia, Cedam, Padova, 2003.

Coda V., Responsabilità sociale e strategica dell'impresa, in Sacconi G., Responsabilità sociale e governo d'impresa. Problemi, teorie e applicazioni. Un esame critico, Maggioli, Rimini. 2005.

Comite U., Creazione di valore e sussidiarietà nell'amministrazione pubblica: un approccio economico-aziendale in G.P. Calabrò (a cura di), La nozione di sussidiarietà tra teoria e prassi, Edizioni Scientifiche Italiane, Napoli, 2009.

Corno F., L'etica nel governo dell'impresa. Convergenza tra pensiero laico e Dottrina Sociale, Guerini e Associati, Milano, 2002.

Di Giandomenico M., Il bilancio sociale e il modulo aziendale etico, Giuffr è, Milano, 2008.

Di Toro P., Governance, etica e controllo, Cedam, Padova, 2000.

Donati P., Colozzi I. (a cura di), La sussidiarietà: che cos'è e come funziona, Carocci, Roma, 2005.

Enciclica Rerum Novarum (papa Leone XIII -1891).

Enciclica Quadragesimo Anno (papa Pio XI - 1931).

Enciclica Centesimus Annus (papa Giovanni Paolo II - 1991).

Enciclica Caritas in Veritate (papa Benedetto XVI - 2009).

European Multistakeholder forum on CSR-final risults and recommendations, 29 giugno 2004-Final Report: 4.

Farneti G., in AA.VV., Il business alla ricerca dell'etica. Fondazioni di impresa e responsabilità sociale delle aziende. Atti del Convegno. Philanthropy Centro Studi, Facoltà di Economia, Forlì 2004.

Gregoratti C., Prospettive critiche in materia di RSI e sviluppo. Una risposta, University of Manchester, 2006.

Muirhead S. A., Philanthropy and Business: The Changing Agenda, The Conference Board, 2006.

Matacena A., L'accountability nelle imprese lucrative e sociali. Verso una possibile convergenza?, Intervento al convegno: La responsabilità sociale delle imprese tra evoluzione scientifica e processi attuativi, Catania: 8-9 settembre 2005.

Millon -Delsol C., Il principio di sussidiarietà, Giuffrè, Milano, 2003.

Molteni M., Responsabilità sociale dell'impresa. Verso la sintesi sociocompetitiva, Vita e Pensiero, Milano, 2004. 
Payne D.M., Raiborn C.A., Sustainable Development: The ethics support the economics, Journal of Business Ethics, 32, 2001.

Ponzanelli G., Impresa e ambiente, Circolo giuridico dell'università, Siena, 1963.

Rusconi G., Il bilancio sociale. Economia,etica e responsabilità d'impresa, Ediesse, Roma, 2006.

Terzani S., Responsabilità sociale dell'azienda, Rivista Italiana di Ragioneria ed Economia Aziendale n`7-8, Roma, 1984.

Zadek S., Balancing performance, ethics, and accountability, Journal of business ethics, 17, 1998.

Zadra G., Il rendiconto agli stakeholder. Una guida per le banche, http://www.abi.it/doc/home/conoscereBanche/FinanzaEtica/doc/tmp1 163169967596_30 Rendiconto.pdf.

Zamagni S., Responsabilità Sociale delle Imprese e Democratic Stakeholding, Working paper n²8-2006, Facoltà di Economia, Università di Bologna-Forlì. 